\section{TUBERCULOSIS OF THE BREAST}

BY

\author{
RONALD W. RAVEN, O.B.E., F.R.C.S. \\ Surgeon to Westminster Hospital (Gordon Hospital) and to the \\ Royal Cancer Hospital
}

It appears from a survey of the literature that little attention has been paid during recent years to this subject in English writings. The opportunity is taken, therefore, in presenting this case record, to review the general features of the disease and stress its importance in the differential diagnosis of 'esions of the breast. It is important to appreciate the close similarity between certain forms of tuberculosis and carcinoma of the breast, and differentiation is often rendered difficult; many of the cases of primary tuberculosis reported in the literature were diagnosed incorrectly as carcinoma, and it was only after operation and pathological study of the tissues that the true situation was disclosed.

\section{Case Report}

A woman aged 43 developed a swelling in the left breast in May, 1947, following a blow through a fall one month previously. The swelling was regarded as mastitis of the upper and inner quadrant. In September the left breast became red and tense, and an abscess was incised and drained. Pathological investigation of the tissues showed chronic inflammation with no giant-cell systems or tubercle bacilli. After the operation a small sinus persisted, with slight discharge. On January 6, 1949, a small red swelling developed in the upper and outer quadrant of the left breast, and this became cystic and tender. Aspiration yielded $1.5 \mathrm{ml}$. of purulent fluid which showed tubercle bacilli on microscopy.

She had had amenorrhoea between May, 1947, and February, 1948 - before this the cycle was 28 days and the duration five days - and again since April, 1948. She had never been pregnant. Her general health was good; no cough or pain in the chest. When 4 years old she had had tuberculous peritonitis, with operative treatment. In December, 1914, an operation had been performed on the left cervical lymph nodes. One brother had had tuberculous cervical lymphadenitis as a child.

On examination the patient looked healthy, Her neck showed scarring on the left side; no enlarged lymph nodes. The heart and lungs showed no gross abnormality. Scars of previous operations were seen in the right hypochondrium, but no other abdominal abnormality was found. The right breast was normal. In the lower half of the left breast there was a discharging sinus with an associated abscess ; a red fluctuating tender swelling was seen in the upper and outer quadrant (see Fig.) No marked induration of the breast tissue was present. The lymph nodes in the axillae were not enlarged. $X$-ray examination of the chest revealed no abnormality.

Photograph of the affected breast showing the tuberculous lesion in the upper and outer quadrant. There is an area of puckering in the lower and inner quadrant.

Operation. - Local mastectomy and excision of the fourth costo-chondral junction, left side. It wás noted that the sinus in the lower part of the breast was connected with a caseous process in the fourth costo-chondral junction, left. The wound healed by first intention.
Study of Specimen.-In the left breast there was an abscess situated above and external to the nipple. The skin over the abscess was bluish in colour. On opening into the abscess cavity it was seen to contain yellow caseous material. In the lower and inner quadrant of the breast there was an area of puckered scar tissue. Sections through the breast beneath this scar tissue showed a sinus which could be traced through the substance of the breast to its deepest part. This sinus contained caseous material and was presumably connected with the rib which was resected. A piece of resected rib was partly replaced at the costo-chondral end by an irregular mass of granulation tissue. On histological examination both the wall of the abscess cavity and the sinus showed tubercles composed of endothelial cells surrounding areas of caseation. There was also a small round-cell infiltration. Breast tuberculosis was diagnosed.

\section{General Features of the Disease}

The condition was described in the first instance by Sir Astley Cooper, who wrote in 1829: "In young women, who have enlargement of the cervical absorbent glands, I have sometimes, though rarely, seen tumours of a scrofulous nature form in their bosoms, confined in most cases to a single tumour in one breast; but in one case, two existed in one breast, and one in the other. They are entirely unattended with pain, are distinctly circumscribed, are very smooth on their surfaces, and scarcely tender to pressure. They are very indolent, and vary with the state of the constitution, diminishing as it improves, and increasing as the general health is deteriorating." He pointed out that the condition does not degenerate into malignancy, and surgical treatment is contraindicated. His concluding remarks were: "It will be admitted by every one who deserves the title of a surgeon, that we possess no specific remedy for this disease, but that we are required to assist the digestive powers, make better blood; and convey it to the system by an increased vigour of the constitution." Since this publication over 500 cases have been reported in the literature.

The incidence has been computed at various figures: thus, Nicolson and Gillespie (1941) consider the incidence of tuberculosis compared with all breast lesions to be $0.54 \%$; McGehee and Schmeisser (1935) give a figure of $1.87 \%$. On this basis it is not a common condition, but it is possible that a number of cases are overlooked, the rarity being apparent rather than real.

There are two main varieties-namely, primary tuberculosis, in which there is no evidence of a tuberculous lesion elsewhere in the body, and secondary tuberculosis, in which there is evidence of a tuberculous lesion elsewhere, such as in the lungs, pleura, lymph nodes, or bone. The various lesions can be grouped under three headings as follows:

Nodular Lesion.-A circumscribed lump of variable size forms in the breast, slow caseation occurs with softening of the tissues, and eventually an abscess forms which may produce a sinus in the overlying skin.

Sclerosing Lesion.-A circumscribed lump develops which is hard in consistency owing to excessive fibrous-tissue formation. This variety is seen in older patients.

Atypical Lesions.-A tuberculous ulcer develops in the skin near the nipple and nodules are formed in the breast tissue later; a subcutaneous lesion develops which resembles a furuncle ; or vesiculated pimples form with indurated bases.

\section{Mode of Entry of Tubercle Bacillus}

Considerable discussion has been aroused regarding the possible routes of entry. The organisms may gain an entrance through the ducts of the nipple or through an abrasion in the skin, but these must be remote possibilities, although several cases have been reported. Thus, Morgen (1931) quotes Ingier, who described a case in which the inflammatory process was confined to the walls of the excretory ducts and the periacinous tissue, the interacinous tissue 
being only slightly involved. Morgen also quotes Ely, who mentions a woman who covered her breast with a piece of linen contaminated with tuberculous sputum and soon after developed tuberculosis in this breast.

Haematogenous infection may occur, although Nagashima (1925) investigated 34 patients who died of miliary tuberculosis, studying the breast tissue microscopically, by smears, and guinea-pig inoculations, but was unable to demonstrate any involvement. The case recorded above is instructive in that multiple tuberculous lesions developed at long intervals in widely separated parts of the body, and it is assumed the tubercle bacilli were transported by the blood stream.

Many authors have stressed the importance of infection by the lymphatic system ; the primary lesion being cervical, axillary, or mediastinal tuberculous adenitis, and the breast being affected by retrograde infection. Morgen quotes Halsted and Le Court, who state that there is tuberculous axillary adenitis on the same side as the affected breast in $75 \%$ of cases, and Berchtold, who found the primary disease in the axillary lymph nodes and the secondary in the breast in 8 out of 37 cases reported. He also calls attention to a case described by Johannet in which at necropsy subpleural tuberculosis was demonstrated, extending to the axillary lymph nodes and eventually to the breast.

Finally, the breast may be involved from underlying tuberculosis of the sternum, costo-chondral junction, or rib. In this case the lower of the two lesions extended directly down to a diseased costo-chondral junction.

\section{Symptomatology and Differential Diagnosis}

The disease occurs between the ages of 20 and 50, when the breasts are most active. The majority of patients are apparently in good health; some may give a past history of tuberculosis, as did my patient, who had had tuberculous peritonitis and cervical adenitis. The most frequent presenting symptom is a lump in the breast, which is often painless and discrete.

When caseation is present the border of the lump is well defined. The overlying skin shows certain changes; thus there is often fixation and dimpling with retraction of the nipple, or it is stretched and red, sinus formation developing later. Retraction of the nipple may be the first sign of the disease. An abscess is present sometimes, and Morgen states that a fistula is seen in $50 \%$ of cases. Tubercle bacilli may be demonstrated in the discharge. Other lesions may be present in the breast, including fibro-adenoma or carcinoma.

Enlargement of the axillary lymph nodes is often present, and this may precede the development of the disease in the breast. A radiological examination of the chest should be made in every case; Grausman and Goldman (1945) state that pulmonary tuberculosis is present in 12 to $16 \%$ of cases. The disease is usually unilateral, but cases with both breasts involved have been reported.

The following diseases must be considered in the differential diagnosis.

Carcinoma.-Many of the reported cases of tuberculosis of the breast were misdiagnosed as carcinoma, and differentiation may be difficult, especially in the sclerosing types. The degree of hardness is greater in carcinoma and the axillary lymph nodes may also be hard. The deformity of the nipple may be greater in carcinoma. Tuberculosis tends to occur in younger age groups than carcinoma, and there may be a purulent discharge from the nipple. When a sinus is present it may be possible to isolate the tubercle bacillus; in all doubtful cases a biopsy and histological examination must be performed.
Benign Fibro-epithelial Tumours.-Differentiation is not difficult usually in these cases. The tumours are circumscribed and encapsulated and mobile in the breast tissue. There is no involvement of the overlying skin nor any change in the nipple. The axillary lymph nodes are not enlarged.

Cystic Hyperplasia.-In this condition both breasts are often involved and the swellings produced are larger. The cysts are palpable, causing an uneven surface in the swelling. There are no changes in the overlying skin. The axillary lymph nodes are not enlarged.

Acute Mastitis.-Differentiation is not difficult in this instance. There are fever and other general symptoms of an acute infection, and locally the cellulitis, with or without abscess formation, is typical of the condition.

Actinomycosis.-When a discharging sinus is present, together with an indurated swelling in the breast, actinomycosis must be considered, although this is an uncommon lesion. The typical sulphur granules may be present in the discharge and the fungus isolated.

Gumma.-This is an uncommon lesion in the breast; degeneration may occur with sinus formation in the skin. A Wassermann test and biopsy will help in the diagnosis.

Traumatic Fat Necrosis. - There may be a history of an injury to the breast, causing a localized hard swelling without any inflammatory signs. Biopsy and microscopical examination may be required to determine the diagnosis.

\section{Association of Tuberculosis with Carcinoma of Breast}

The presence of tuberculosis and carcinoma in the same breast has been noted on a number of occasions. Thus, Grausman and Goldman (1945) collected 26 cases from the literature and added two cases of their own. It is obviously important to remember this possibility, as an associated carcinoma may be overlooked, with serious consequences for the patient. Morgen discussed the interpretation of the relationship of these conditions. The two conditions may occur independently, either simultaneously or consecutively. The tuberculosis may be engrafted on the malignant condition as a result of the greater susceptibility of breaking-down tissue to infection. It is possible that the carcinoma is the result of the chronic irritation of the tuberculous inflammatory process.

In a number of these cases it was thought that the carcinoma was of longer duration in the breast and of a fibrous type; the tuberculous lesions showed no calcification, so it is possible that tuberculous infection occurred in tissues of decreased resistance or increased susceptibility. In some cases of carcinoma of the breast it was found at operation that there was tuberculous adenitis in the axillary lymph nodes. The breast is not the only site where these conditions coexist, for carcinoma has been found in chronic tuberculous cavities in the lungs.

\section{Treatment}

The maintenance of the patient's general health is important, as in all cases of tuberculosis. When the disease is localized to the breast and there is no evidence of involvement of the axillary lymph nodes, local mastectomy should be performed. If there is enlargement of the axillary lymph nodes mastectomy is combined with excision of these lymph nodes, and a careful dissection of the axilla is necessary. In order to carry this out the tendons of the pectoralis major and pectoralis minor muscles should be divided to open up the axilla; at the end of the dissection the tendon of the former muscle is sutured. If the underlying costochondral junction or rib is involved, this must be excised. When tuberculosis and carcinoma coexist a radical mastectomy is performed with or without high-voltage $x$-irradiation, according to the type of carcinoma which is present. Now that streptomycin is available in greater quantities its action in cases of tuberculous mastitis may be beneficial, 
and before resorting to surgery in the future the effects of this drug should be tried.

\section{Summary}

A case record is presented of tuberculosis of the breast and underlying costo-chondral junction. The patient gave a history of old tuberculous peritonitis and tuberculous cervical adenitis. Tubercle bacilli were isolated from the discharging sinus. The general features of the disease, the symptomatology, and the differential diagnosis are described, and special emphasis is laid on the importance of differentiating carcinoma. Attention is called to the association of tuberculosis and carcinoma in the same breast. The treatment is described for various types of case.

I wish to thank Dr. J. H. O. Earle for studying the pathology of the specimen, and Dr. Peter Hansell for the photograph.

\section{REFERENCES}

Cooper, Sir Astley (1829). Illustration of Disease of the Breast. London.

Grausman, R. I., and Goldman, M. L. (1945). Amer. J. Surg., 67, 48.

McGehee, J. L., and Schmeisser, H. C. (1935). Ibid., 28, 461

Morgen, M. (1931). Surg. Gynec. Obstet., 53, 593.

Nagashima, Y. (1925). Arch. Path. Anat.. 254, 184.

Nicolson, W. P., and Gillespie, C. E. (1941). Sth. Surg., 10, 825.

\section{SOLITARY CEREBRAL METASTASES FROM BRONCHIAL CARCINOMATA \\ THEIR INCIDENCE AND A CASE OF SUCCESSFUL REMOVAL}

BY

\section{GEOFFREY FLAVELL，F.R.C.S., M.R.C.P.}

(From the Department of Thoracic Surgery, London Hospital)

The extirpation of metastases is not generally regarded as a rewarding surgical practice, and although many an abdominal surgeon must have been tempted to resect an apparently single deposit in the liver, and many a thoracic one must at some time or other have mistaken a secondary tumour in the lung for a primary lesion, few records of their experiences exist, and fewer still of the planned removal of both primary and secondary growths.

One is apt to assume that the appearance of a metastasis inevitably implies the existence of others and the certainty of death, and examples in the literature to support a contrary hope are rare. In 1946 Alexander and Haight were, however, able to collect the histories of 24 patients who had undergone pulmonary resections for solitary metastases in the lung after previous excisions of the primary growths, 15 of which were carcinomata and eight sarcomata. Of the 24, one died, 11 had recurrences, but 12 remained free ; and among the latter eight survived their resections by more than a year. One patient (who lived 12 years) had originally been operated upon by Barney (1944a, 1944b) for a renal adenocarcinoma, the lung metastasis having been seen a year before his nephrectomy and removed a year later, so that its malignancy must have been low. Six other of the primary lesions were also renal-cell carcinomata, and it is of interest that both Bumpus (1928) and Beer (1937) have reported spontaneous regression of such metastases.

Albright in 1944, Friedman in 1943, and Turner and German in 1941 have described in all four instances of successful extirpation of characteristically slow-growing skull metastases from thyroid carcinomata; and Cattell (1940) reports a patient well one year after the removal of a solitary secondary in the liver at the abdominal stage of an abdomino-perineal resection of a rectal carcinoma. He argues, "The patient is better off with a large focus of malignancy removed, and there is at least some clinical evidence that there may be retardation of the malignant process."

The only successful removal on record (apart from the one reported below) of both primary and cerebral secondary growths was carried out by Thurel in 1942, when he excised a deposit from the right frontal lobe of a patient who had had a Halsted operation for carcinoma of the breast exactly a year previously. She remained symptomless two years later (Alajouanine, Thurel, and Longuet, 1944).

The incidence of cerebral metastasis in carcinoma of the lung was estimated by Ochsner and de Bakey, reviewing 3,047 patients, to be $16.5 \%$. Other authorities put the figure even higher-Graham and Wagner at $24 \%$ and Olsen at $36 \%$. In 448 proved pulmonary cancers Tinney and Moersch (1944) found that $19(4 \%)$ presented with central nervous symptoms, and in these people the average duration of life from the onset of their symptoms was six months.

In the necropsy records of the London Hospital from 1909 to 1949 I have been able to find 85 examples of cerebral metastases accompanying a primary bronchial carcinoma. Of these, 26 were single in the brain or cerebellum-a percentage of 31 in a group of whom many died in the extremity of their disease, with numerous secondary deposits elsewhere in their bodies. Furthermore, eight ( $9.4 \%$ ) were completely solitary in the body (if removable hilar glands alone be ignored); the cerebral lesion was a lonely satellite of the pulmonary primary growthone, indeed, occurred two years after a successful pneumonectomy.

\section{Case Report}

In June, 1947 , a man aged 34 , in a responsible clerical position, was found on mass radiography to have a circumscribed shadow at the hilum of his left lung. He had had no symptoms of any sort, but one day in October he developed a headache, accompanied by noises and followed an hour later by an epileptiform fit in which he lost consciousness for 10 minutes and was incontinent of urine. This was not repeated, but frontal and vertical headaches persisted and were later associated with occasional diplopia, flashes of light, and other visual disturbances. He was admitted to the London Hospital on November 22, 1947, and except for early papilloedema, very slight left lower facial weakness, and equally slight bilateral hypotonia (a little more pronounced upon the left side), he had no abnormal physical signs either of his chest or of his nervous system. Lumbar puncture revealed a pressure of $210 \mathrm{~mm}$., and the protein content of the cerebrospinal fluid was raised to $120 \mathrm{mg}$. per $100 \mathrm{ml}$. Although bronchoscopy was negative, there seemed to be little doubt that a primary carcinoma of the left lung was accompanied by a cerebral metastasis. Since the necropsy findings on similar patients, as already mentioned, gave some encouragement, a deliberate plan was then made to attack the secondary deposit, and, if it proved operable and was single, subsequently to remove the lung with its primary growth.

Because the symptoms and signs of raised intracranial pressure became severe, a palliative right subtemporal decompression was performed on December 18. This proved effective, and on January 8,1948 , ventriculography showed displacement of the system to the left, with no filling of the right ventricle, indicating a large right-sided lesion. Consequently a right fronto-lateral exploratory craniotomy was carried out by Mr. D. W. C. Northfield; this revealed a highly vascular lobulated mass about 7 by $4 \mathrm{~cm}$., occupying a superficial position in the right temporal lobe, but not transgressing the Sylvian fissure. It was secondarily adherent to the dura mater over some $2 \mathrm{~cm}^{2}$ The whole tumour, with a narrow margin of apparently healthy brain tissue, was removed with the overlying adherent dura. Histological section proved it to be a secondary carcinoma, but gave little indication of its origin

The patient made a good recovery, and eight weeks later was readmitted, this time to the thoracic surgery department. 